\section{An introduction to biosimilar cancer therapeutics: definitions, rationale for development and regulatory requirements}

\author{
Christian Buske ${ }^{*, 1}$, Michinori Ogura ${ }^{2}$, Hyuk-Chan Kwon ${ }^{3} \&$ Sang Wook Yoon ${ }^{3}$
}

\begin{abstract}
Monoclonal antibodies and other biologic drugs play important roles in the treatment of various hematological malignancies and solid tumors. However, such drugs are intrinsically more expensive to develop than small molecules and their clinical benefits are often accompanied by challenges relating to affordability and access. Patent expiry for 'originator' biologics is providing opportunities for a new generation of biosimilar drugs, potentially capable of relieving pressure on healthcare budgets. This article discusses key characteristics of biosimilars, distinguishes them from generics and noncomparable biologics and outlines the robust regulatory requirements that must be followed to establish biosimilarity with a reference product. The path to approval is discussed with reference to the rituximab biosimilar CT-P10, the first licensed monoclonal antibody biosimilar cancer therapeutic.
\end{abstract}

First draft submitted: 30 March 2017; Accepted for publication: 20 April 2017; Published online: 9 May 2017

Targeted biologic therapies have had a marked impact on clinical practice and patient outcomes in several areas of medicine over the past two decades, perhaps most notably in rheumatology, hematology and oncology. For example, various monoclonal antibodies (mAbs) - including TNF inhibitors, such as infliximab in rheumatoid arthritis (RA), rituximab in CD20-positive hematological malignancies and trastuzumab in HER2-positive solid tumors - are now considered integral components of standard care after their beneficial effects on symptoms, disease progression and/or survival were demonstrated, first in clinical trials and then in the clinic [1-4].

$\mathrm{mAbs}$ and other biologic therapies are highly complex, large molecules that are made in living systems and require considerable scientific expertise and monetary investment to develop and manufacture. Partly as a result of these factors, the costs of these drugs are often high and patient access to them frequently limited [5]. In recent years, however, the actual or pending expiry of patents for various targeted drugs - in addition to demands from patients and other stakeholders for more costeffective treatments and greater access - has led to the development of cheaper, biosimilar drugs, including those used to treat cancer. The potential impact of such 'biosimilar cancer therapeutics' is reflected by their prominence as major topics of discussion among oncologists and hematologists at recent global congresses [6,7].

The recent approval in Europe and South Korea of CT-P10 (Truxima ${ }^{\circledR}$; CELLTRION, Incheon, Republic of Korea) has heralded the imminent arrival of biosimilar cancer therapeutics in the clinic [8]. CT-P10 is a biosimilar of rituximab, a mAb targeted against the CD20 transmembrane protein found

'CCC Ulm, University Hospital UIm, Ulm, Germany

2Department of Hematology, Tokai Central Hospital, Gifu, Kakamigahara, Japan

${ }^{3}$ CELLTRION Healthcare Co. Ltd, Incheon, Republic of Korea

*Author for correspondence: Tel.: +49 731500 65888; Fax:+49 731500 65822; christian.buske@uni-ulm.de

\section{KEYWORDS}

- biosimilars • chronic lymphocytic leukemia $\bullet$ cost savings $\bullet$ CT-P10 $\bullet$ diffuse large B cell lymphoma - follicular lymphoma - non-Hodgkin's lymphoma - rituximab 
primarily on B cells. Approval of CT-P10 came approximately 20 years after originator or 'reference' rituximab was first approved in the USA in 1997 (as Rituxan ${ }^{\circledR}$; Genentech, CA, USA) and in Europe in 1998 (as MabThera ${ }^{\circledR}$; Roche, Basel, Switzerland). In preparation for the addition of CT-P10 and other new biosimilars to the treatment landscape, this article provides oncologists, hematologists and others involved in the care of patients with cancer with a general introduction to biosimilars and the concept of biosimilarity. A more in-depth consideration of the rationale for development of, and regulatory requirements for, biosimilar cancer therapeutics is also provided. Finally, data on CT-P10 are reviewed as a case study in development of this emerging class of new drugs.

\section{Biosimilars: definitions \& differences versus generic drugs}

A 'biosimilar' has been defined by the EMA as "a biological medicinal product that contains a version of the active substance of an already authorized original biological medicinal product (reference medicinal product)" for which "similarity to the reference medicinal product in terms of quality characteristics, biological activity, safety and efficacy based on a comprehensive comparability exercise needs to be established" [9]. In keeping with the EMA's definition, the US FDA consider 'biosimilarity' to mean that "the biological product is highly similar to the reference product notwithstanding minor differences in clinically inactive components and that there are no clinically meaning ful differences between the biological product and the reference product in terms of safety, purity and potency" [10]. The reference product to which a candidate biosimilar is compared may also be called the 'innovator' or 'originator' drug.

Because biosimilars are approved after expiry of innovator drug patents and are usually less expensive than their reference products, they are sometimes deemed to be essentially the same as generic versions of 'traditional', nonbiologic drugs. However, biosimilars differ from generic drugs in several important ways (Figure 1). Most notably, generics are small-molecule drugs of precisely known structure that are synthesized by predictable and easy-to-replicate chemical reactions, with these reactions yielding exact copies of their reference products [11,12]. In contrast, biosimilars - often large, complex proteins containing amino acids and numerous glycan structures - are generated in living cell lines $[12,13]$. Due to the natural variation in these molecules, as well as heterogeneity in product processes, it is impossible to develop biosimilars that are identical to their reference product, or indeed identical batches of any individual biologic drug. Factors contributing to the inherent heterogeneity of biologics include variations in the nature and extent of any post-translational modifications, particularly glycosylation, as well as differences in levels of product aggregation and host cell-related impurities [13,14].

Given the expected differences between a biosimilar and its reference product, regulatory requirements for biosimilars are markedly different to those for generic drugs, which are primarily based on establishing product quality and bioequivalence to the reference-branded drug [15-17]. In order to secure approval, a generic drug must have the same qualitative and quantitative composition of active substance(s) as the reference product, and the same pharmaceutical form. In addition, bioequivalence with the reference medicinal product must be demonstrated in appropriate bioavailability studies comparing pharmacokinetic parameters including maximum plasma concentration $\left(\mathrm{C}_{\max }\right)$ and area under the plasma concentration-time curve (AUC) [15-17]. Efficacy and safety of a generic drug need not be investigated as these are presumed to be the same as for the reference product.

It is also important to distinguish 'true' biosimilars, which have undergone rigorous examination versus their reference products, from other versions of originator drugs sometimes referred to as 'biomimics', 'intended copies' or 'noncomparable biologics' (Figure 1). The latter are copies of licensed biological products that have not met the requirements for biosimilarity stipulated by the EMA, FDA or other similarly stringent bodies, and typically originate from countries with nonstringent regulatory pathways $[18,19]$. These products are not, and should not be considered to be, 'biosimilars'. Often, clinical data may be missing or not permit proper comparison against the licensed reference product, potentially resulting in clinically significant differences in product formulation, dosage, quality, efficacy and safety [19]. Such differences may result in less favorable clinical outcomes being achieved with noncomparable biologics versus biosimilars, including diminished therapeutic effectiveness and an increased risk to patient safety. Co-existence of noncomparator 


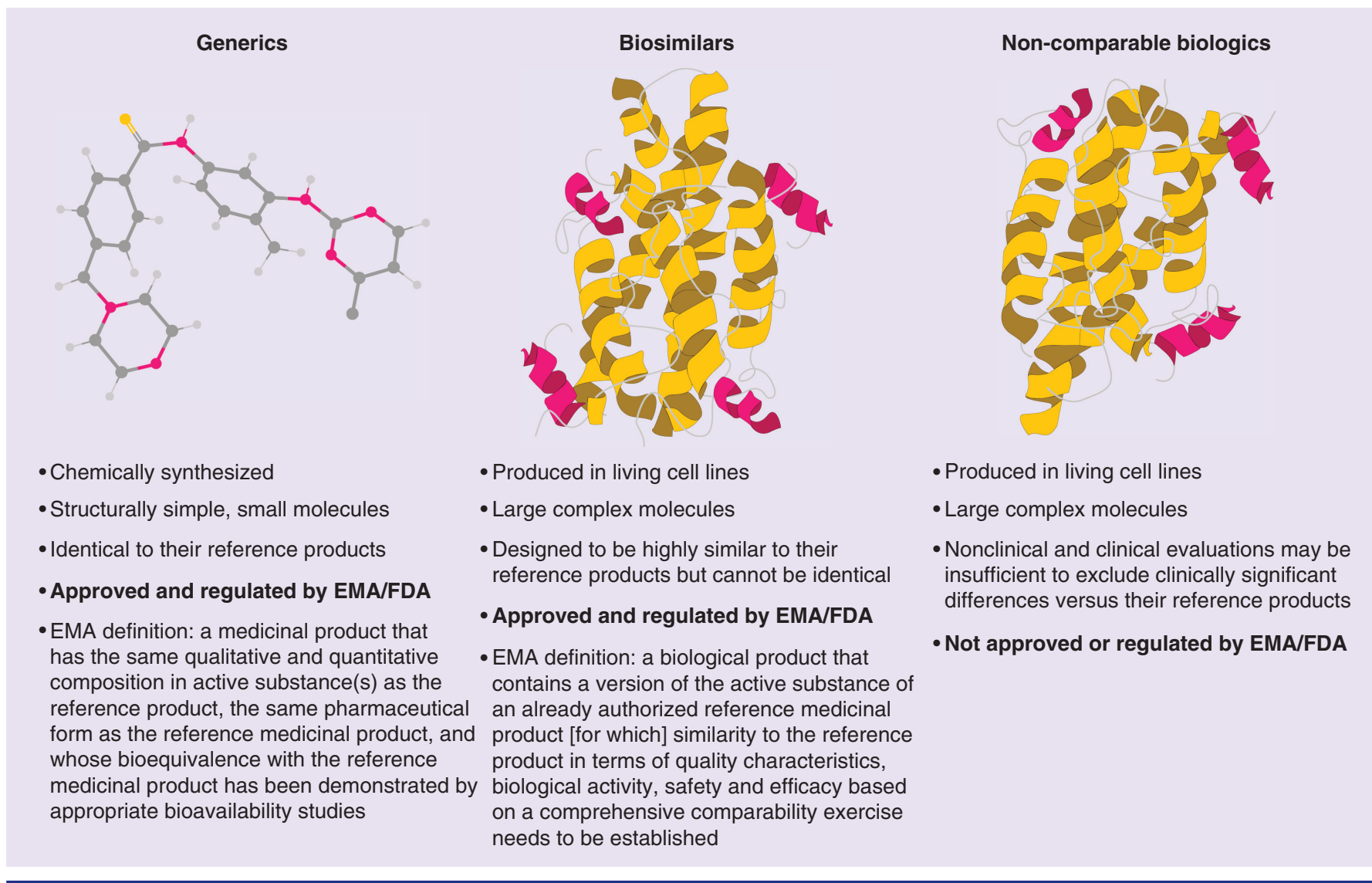

Figure 1. Comparison of generics, biosimilars and noncomparable biologics.

biologics and biosimilars in the market may cause confusion for physicians, other prescribers, pharmacists, payers and indeed for patients [18]. For example, it is possible to hypothesize that safety or efficacy issues arising with the use of a noncomparable biologic that is essentially marketed as a biosimilar could impact on clinician trust in true biosimilars developed according to accepted standards.

In Europe and elsewhere, biosimilars of relatively simple, low molecular weight proteins entered clinical practice from 2006 onwards [20]. These 'first-generation' biosimilars include versions of reference products used in oncology supportive care to counteract or prevent the side effects of chemotherapy, including the G-CSF filgrastim (administered to reduce the incidence and duration of neutropenia) and the erythropoiesis-stimulating agent epoetin alfa (prescribed to treat anemia and reduce the requirement for blood transfusion) [21,22]. In the USA, filgrastim-sndz (Zarxio ${ }^{\oplus}$; Sandoz, Holzkirchen, Germany) became the first biosimilar to be approved by FDA in 2015 [23]. Biosimilars of
$\mathrm{mAbs}$ and other larger, more complex biologics may be considered 'second-generation' biosimilars, their mechanisms of action allowing them to be employed as disease treatments rather than as supportive agents. The first of the second-generation biosimilars to be approved in Europe and the USA (in 2013 and 2016, respectively) was the infliximab biosimilar CT-P13 (infliximabdyyb; Remsima ${ }^{\circledR}$ [CELLTRION], Inflectra ${ }^{\circledR}$ [Pfizer, NY, USA]). CT-P13 is used to treat RA and other immune-mediated inflammatory diseases (namely psoriasis and psoriatic arthritis, ankylosing spondylitis and inflammatory bowel disease) [24,25]. Table 1 lists the biosimilars that are currently approved in Europe and the USA.

\section{Rationale for development \& introduction} of biosimilar cancer therapeutics

Cancer imposes the greatest economic burden on healthcare systems of any cause of death worldwide [27]. The ever-increasing costs of cancer are unsustainable and can prevent some patients from receiving the optimal treatment at the right point in time. The latest statistics 
SUPPLEMENT Buske, Ogura, Kwon \& Yoon

Table 1. Biosimilars currently approved in Europe (by the EMA) and in the USA (by the FDA).

\begin{tabular}{|c|c|c|c|}
\hline Reference product & Active substance & Biosimilar & Year of approval \\
\hline \multicolumn{4}{|c|}{ EMA-approved biosimilars } \\
\hline \multirow[t]{2}{*}{ Clexane } & Enoxaparin sodium & Inhixa & 2016 \\
\hline & & Thorinane & 2016 \\
\hline Enbrel & Etanercept & Benepali & 2016 \\
\hline \multirow[t]{5}{*}{ Eprex/Erypo } & Epoetin alfa & Abseamed & 2007 \\
\hline & & Binocrit & 2007 \\
\hline & & Epoetin alfa Hexal & 2007 \\
\hline & Epoetin zeta & Retacrit & 2007 \\
\hline & & Silapo & 2007 \\
\hline \multirow[t]{2}{*}{ Forsteo } & Teriparatide & Movymia & CHMP positive opinion 10 November 2016 \\
\hline & & Terrosa & 2017 \\
\hline Genotropin & Somatropin & Omnitrope & 2006 \\
\hline \multirow[t]{2}{*}{ Gonal-f } & Follitropin alfa & Bemfola & 2014 \\
\hline & & Ovaleap & 2013 \\
\hline \multirow[t]{2}{*}{ Humira } & Adalimumab & Amgevita & 2017 \\
\hline & & Solymbic & CHMP positive opinion 26 January 2017 \\
\hline \multirow[t]{2}{*}{ Lantus } & Insulin glargine & Abasaglar & 2014 \\
\hline & & Lusduna & 2017 \\
\hline Mabthera & Rituximab & Truxima & 2017 \\
\hline \multirow[t]{8}{*}{ Neupogen } & Filgrastim & Accofil & 2014 \\
\hline & & Biograstim & 2008 \\
\hline & & Filgrastim Hexal & 2009 \\
\hline & & Grastofil & 2013 \\
\hline & & Nivestim & 2010 \\
\hline & & Ratiograstim & 2008 \\
\hline & & Tevagrastim & 2008 \\
\hline & & Zarzio & 2009 \\
\hline \multirow[t]{3}{*}{ Remicade } & Infliximab & Flixabi & 2016 \\
\hline & & Inflectra & 2013 \\
\hline & & Remsima & 2013 \\
\hline \multicolumn{4}{|c|}{ FDA-approved biosimilars } \\
\hline Enbrel & Etanercept & Erelzi & 2016 \\
\hline Humira & Adalimumab & Amjevita & 2016 \\
\hline Neupogen & Filgrastim & Zarxio & 2015 \\
\hline Remicade & Infliximab & Inflectra & 2016 \\
\hline
\end{tabular}

from the WHO reveal an incidence of 14 million cancer cases per year globally, projected to increase to more than 21 million by 2030 [28]. This trend is due in large part to an aging population. Notably, the incidence of cancer is nearly tenfold higher in individuals aged over 65 years, such that treatment of the elderly contributes disproportionately to healthcare costs [29]. Rising expenditure also stems from extended time on treatment due to the increases in survival that have accompanied improvements in diagnosis, treatment and care [30]. In fact, the global cost of cancer therapeutics and supportive care drugs grew to $\$ 107$ billion in 2015 [31], with an estimated $35 \%$ of current expenditure attributable to targeted biologic drugs, specifically mAbs [32] . High-cost drugs such as anticancer innovator biologics are often subject to rationing [29]. While rationing strategies include capping the number of treatments or targeting specific patient populations, sometimes criteria applied appear arbitrary; furthermore, geographical inequalities in access can be marked, even at the regional level [29,33-35].

The higher costs of development and manufacturing for biosimilars compared with generic 
drugs mean that discounts versus the reference product price are usually lower than the very large reductions in cost that can be achieved with generics. In Europe, most biosimilars are marketed with a discount of around $20-35 \%$ versus the price of their reference products [36]. Nevertheless, the impact of biosimilars on both the costs of treatment and patient access has already been demonstrated with first-generation biosimilars used in oncology supportive care. For example, use of epoetin biosimilars in Europe between the time of their launch and 2014 has led to an estimated $27 \%$ reduction in the price of treatment per day, along with a $16 \%$ increase in average uptake (measured as the volume of treatment days) [37]. In countries where access to epoetins was especially restricted (e.g., Bulgaria, the Czech Republic and Romania), cost savings have been estimated at $50 \%$, with an increase in average uptake of more than $250 \%$ [37].

Looking ahead, cumulative savings of approximately $€ 15$ billion are predicted across five European countries (France, Germany, Italy, Spain and the UK) between 2016 and 2020 , based on a $30 \%$ reduction in price per treatment-day after loss of exclusivity of eight key originator biologics (namely adalimumab, etanercept, follitropin alfa, infliximab, insulin glargine, pegfilgrastim, rituximab and trastuzumab) [37]. When these savings are combined with those from the USA, cumulative savings of $€ 49-98$ billion over this period are predicted [37]. With respect to second-generation biosimilars, budget impact analyses have predicted significant cost savings, which could be diverted in whole or in part to increase treatment access [38]. Recent evidence suggests that such opportunities are driving uptake of CT-P13; in the UK, for example, this biosimilar reached $73 \%$ market share for infliximab by mid-2016 [39].

\section{Scientific rationale \& regulatory requirements for biosimilar cancer therapeutics}

The EMA and FDA have developed specific guidelines for the regulation and approval of candidate biosimilars [9-10,40-42]. The main principles on which these are based are highly aligned, with both pathways involving direct comparison of a candidate biosimilar with its reference product to establish biosimilarity in analytical, in vitro, nonclinical in vivo and clinical studies (Figure 2) [9,10,40-42]. Both agencies advocate a progressive, stepwise approach to the assessment of biosimilarity, with data at each stage of evaluation in part determining the extent and type of assessment required at subsequent steps (e.g., findings in analytical studies may render some in vitro studies appropriate and others unnecessary).

Comprehensive analytical studies form the foundation of comparisons between a candidate biosimilar and its reference product $[9-10,40-42]$. These should include structural analysis and physicochemical characterization. Analytical testing is followed by biochemical and functional in vitro assays, encompassing both the pharmacological target and known pharmacotoxicity of the reference product. Studies should be comparative, reproducible and utilize specific and sensitive assay systems that are carefully selected according to the goals of each analysis. The next step in the evaluation of a candidate biosimilar product is typically nonclinical in vivo studies, which may address outstanding questions from in vitro testing, demonstrate biosimilarity with the reference product with respect to efficacy and/or provide pharmacokinetic and safety data. The EMA and FDA differ somewhat in their stance on in vivo testing. The default position of the FDA is to require in vivo toxicology data; however, the EMA adopts a risk-based approach for all in vivo studies, dependent on in vitro findings. The final phase, clinical evaluation, is itself a step-wise process. The first clinical trial to be carried out is normally a comparative pharmacokinetic study, with the EMA preferring a single-dose, cross-over study incorporating full pharmacokinetic profile characterization. Comparative clinical pharmacodynamic and/or efficacy parameters are also evaluated in clinical trials, as are safety and immunogenicity. Where appropriate, multiple assessments may be conducted within the same study, with this approach helping to compensate for any subtle differences in results arising from the use of different assays or models with different sensitivities or accuracies.

Ultimately, the decision to approve a biosimilar is based on the 'totality of evidence', an approach that integrates data derived from all methods and attributes weight to its quality and quantity. A license will only be granted if the cumulative findings show that no clinically significant differences in efficacy or safety exist between the proposed biosimilar and the reference product $[9,10]$. In addition, biosimilar manufacturers commit to postapproval 


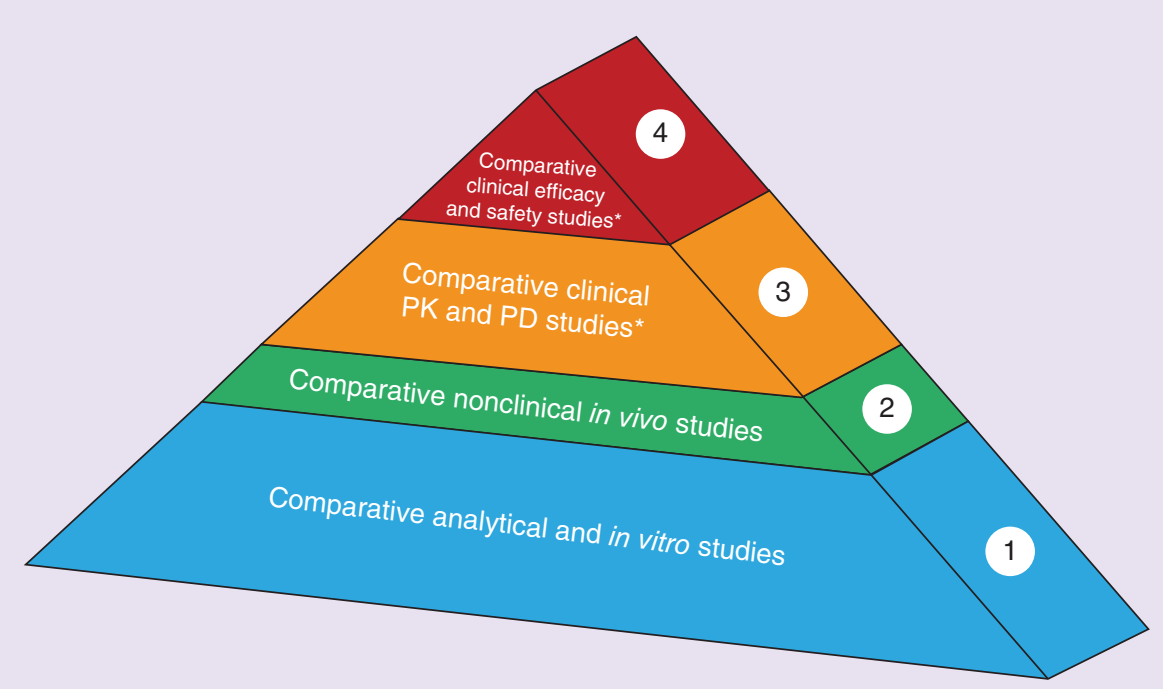

Figure 2. The stepwise approach to biosimilar development, as required by the EMA and FDA. Demonstration of biosimilarity is founded on analytical and in vitro comparisons between a candidate biosimilar and its reference product, followed in some instances by comparative in vivo animal studies. The final components of the 'totality of evidence' for a biosimilar are data from randomized controlled clinical trials directly comparing the PK, PD, efficacy and safety (including immunogenicity) of the biosimilar and reference product.

*Data on PK, PD, efficacy and safety (as well as immunogenicity) are usually gathered in each clinical trial that is performed rather than in a purely stepwise manner.

PD: Pharmacodynamics; PK: Pharmacokinetics.

pharmacovigilance and risk management, such as 'real-life' surveillance studies or postapproval clinical trials, including the monitoring of safety and immunogenicity [9-10,12,36].

Both the EMA and FDA allow indication extrapolation, providing this is scientifically justifiable and is based on the totality of the analytical, in vitro, nonclinical in vivo and clinical evidence. Extrapolation refers to the approval of a biosimilar in one or more additional indications of the reference product, based on the performance of clinical trials in one or more of the most sensitive populations (i.e., populations in which potential differences between the reference product and biosimilar are most likely to be detected). To determine if extrapolation is scientifically justified, consideration is made of various factors, including the mechanisms of action of the reference product in each condition, and the comparability of the biosimilar and reference product in relevant in vitro functional tests $[9,10]$.

Case study: development \& approval of the rituximab biosimilar CT-P10

The rituximab reference product (abbreviated subsequently as 'RTX') was the first mAb to be approved for use in patients with hematological malignancies (or indeed any cancer indication). Since its introduction into clinics in the late 1990s, RTX has become a standard of care in CD20-positive hematological malignancies [2]. RTX is a chimeric mAb consisting of two antigen-binding fragments (Fab) and a crystallizable fragment $(\mathrm{Fc})$. Fab binds to CD20, a surface antigen that is expressed on the surface of both normal and malignant B cells, while Fc binds to Fc $\gamma$ receptors on tumor and immune effector cells [2]. Binding of RTX to CD20 triggers B cell death via three main mechanisms: complement-dependent cytotoxicity (CDC), antibody-dependent cellular cytotoxicity and apoptosis [43].

In February 2017, CT-P10 was approved by the EMA for all indications held by RTX, thus allowing use of this biosimilar in place of RTX in patients with non-Hodgkin's lymphomas (diffuse large B cell lymphoma and follicular lymphoma [FL]), chronic lymphocytic leukemia, RA, granulomatosis with polyangiitis (GPA) and microscopic polyangiitis (MPA). CT-P10, European-sourced RTX (EU-RTX; MabThera) and US-sourced RTX (US-RTX; Rituxan) are identical with respect to pharmaceutical form, 
concentration, composition and method of administration [8]. Consistent with the step-wise approach to establishing biosimilarity advocated by the regulatory authorities, CT-P10 and RTX were compared first in analytical studies followed by in vitro, nonclinical in vivo and clinical studies.

\section{- Analytical \& in vitro studies}

Structural analysis and physicochemical characterization of CT-P10 relative to EU-RTX and US-RTX revealed either high levels of similarity or acceptable differences on all measured parameters [8]. To allow for possible inter-batch variability, testing was performed on multiple batches of each of the three drugs. Primary and higherorder structures were evaluated using validated techniques including peptide mapping, Fourier transform infrared spectroscopy and circular dichroism (for primary, secondary and tertiary structural analyses, respectively). Other measured variables included protein content, purity, charge variants and post-translational glycosylation. All measured variables were highly similar with the exception of some clinically nonsignificant differences in post-translational deamidation, oxidation and C-terminal lysine variants, the relative proportion of charge variants and levels of residual process-related impurities [8].

In vitro biological assays revealed that CT-P10, EU-RTX and US-RTX had highly similar binding affinities for the $\mathrm{CD} 20$ receptor and a range of Fc receptors [8]. For example, the relative binding affinity of CT-P10 to the neonatal Fc receptor $(\mathrm{FcRn})$, which was measured by surface plasmon resonance and calculated as a percentage of the equilibrium dissociation constant $\left(\mathrm{K}_{\mathrm{D}}\right)$ for RTX, was around $100 \%$ [44]. Highly similar potency in eliciting CDC, antibody-dependent cellular cytotoxicity and apoptosis was also demonstrated for CT-P10, EU-RTX and US-RTX in functional analyses in vitro [8]. In the CDC assay, for example, half-maximal effective concentrations $\left(\mathrm{EC}_{50}\right)$ for CT-P10, expressed as a percentage of RTX $\mathrm{EC}_{50}$ values, were approximately $100 \%$ [44].

\section{- Nonclinical in vivo studies}

An in vivo, 8-week toxicokinetic study was performed in cynomolgus monkeys to support assessment of biosimilarity between CT-P10 and EU-RTX [8]. Six animals were included in each of a control, CT-P10 and EU-RTX group. Doses of $20 \mathrm{mg} / \mathrm{kg} /$ week were administered via weekly intravenous (IV) bolus injection. $\mathrm{C}_{\max }$ and AUC values at day 1 were comparable

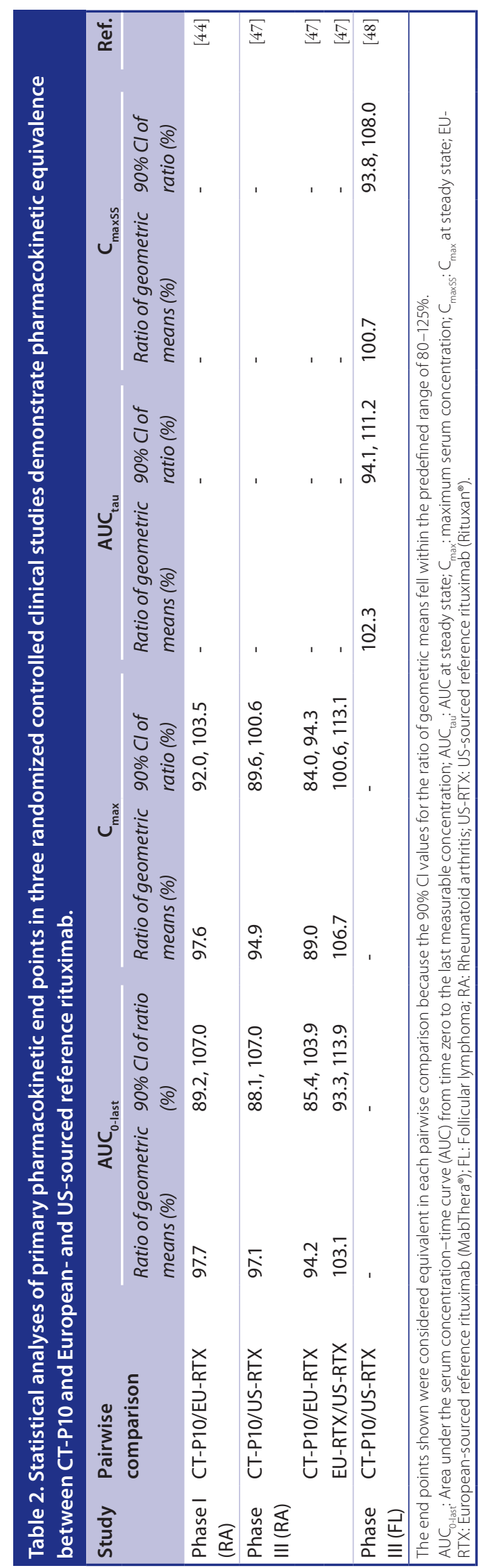


between CT-P10 and EU-RTX. These values were also measured at day 22 although findings at this time point were inconclusive due to the exclusion of some animals following detection of anti-drug antibodies (ADAs) [8].

\section{- Clinical studies}

\section{Pharmacokinetics}

The clinical pharmacokinetics of CT-P10 and its reference product were first compared in patients with active RA [44]. In this Phase I trial [45], 154 patients were randomized 2:1 to receive IV infusions of $1000 \mathrm{mg}$ CT-P10 or EU-RTX at weeks 0 and 2, alongside continued methotrexate therapy. The pharmacokinetic equivalence of the two drugs was proven as the $90 \%$ CIs for the ratios of geometric means (CT-P10/EU-RTX) of the two primary study end points (AUC from time zero to the last measurable concentration $\left[\mathrm{AUC}_{0-\text { last }}\right.$ ] and $\mathrm{C}_{\max }$ after second infusion) fell within the predefined bioequivalence range of $80-125 \%$ (Table 2) [44]. In another study in RA [46], proof of pharmacokinetic equivalence was extended to US-RTX [47]. In the first part of this Phase III trial, 189 patients were randomized 1:1:1 to CT-P10, EU-RTX or US-RTX. The primary end points were $\mathrm{AUC}_{0 \text {-lass }}$, AUC from time zero extrapolated to infinity $\left(\mathrm{AUC}_{0 \text {-inf }}\right)$ and $\mathrm{C}_{\max } ; 90 \%$ CIs for the ratios of geometric means fell within the $80-125 \%$ bioequivalence for each end point and for each pairwise comparison (CT-P10/EU-RTX; CT-P10/US-RTX and US-RTX/EU-RTX) (Table 2) [47].

The pharmacokinetics of CT-P10 have also been evaluated in patients with hematological cancer, specifically those with newly diagnosed, advanced-stage FL who participated in a randomized, controlled, Phase III trial performed in Europe, Africa, Asia Pacific and Latin America [49]. In the first part of this study, 121 patients aged $\geq 18$ years with histologically confirmed FL of grade 1-3a, confirmed CD20positive lymphoma and Ann Arbor Stage III or IV disease were randomized $1: 1$ to receive IV infusions of $375 \mathrm{mg} / \mathrm{m}^{2}$ CT-P10 or US-RTX on day 1 of eight 21-day induction cycles, in combination with cyclophosphamide, vincristine and prednisone (CVP) chemotherapy [48,50]. Analyses of the primary pharmacokinetic end points (steadystate AUC and $\mathrm{C}_{\max }$ at cycle 4) established the pharmacokinetic equivalence of CT-P10 and US-RTX. The $90 \%$ CIs of the ratio of geometric least squares means for both parameters were entirely within the 80-125\% equivalence margin
(Table 2). Mean serum drug concentrations were also highly similar between groups over treatment cycles $1-4$ [48].

\section{Pharmacodynamics}

In the Phase I and III RA studies, rapid and complete depletion of peripheral B cell counts was observed after the first infusion of CT-P10 or RTX $[44,51]$. B cell kinetics up to cycle 4 in the Phase III study of advanced FL has been reported [48]. The median number of B cells fell below the lower limit of quantification $1 \mathrm{~h}$ after the end of the infusion at cycle 1 and remained below this threshold up to cycle 4 .

\section{Efficacy}

Efficacy was comparable between CT-P10 and RTX in the Phase I and III RA studies, as measured using well-validated end points commonly employed in trials involving this patient population. For example, at week 24 of the Phase I study, the proportion of patients achieving American College of Rheumatology (ACR) 20, 50 or $70 \%$ responses - three end points representing reductions in disease activity of increasing magnitude - were highly similar between the two treatment groups (ACR20/50/70: 63.0\%/37.0\%/16.0\% for CT-P10 and $66.7 \% / 31.3 \% / 14.6 \%$ for EU-RTX) [44]. In the Phase III RA study, efficacy (as well as safety and immunogenicity) was assessed in patients who had contributed to the pharmacokinetic analysis described above plus an additional cohort of recruited patients. In total, 372 patients participated in the study and received CT-P10 $(\mathrm{n}=161)$ or RTX (EU- or US-RTX; $\mathrm{n}=211$ ). The adjusted mean change from baseline in the primary efficacy end point (the '28-joint Disease Activity Score using C-reactive protein') was comparable between CT-P10 and RTX groups; 95\% CI for the treatment difference in this change fell entirely within predefined efficacy equivalence margins [51]. Data comparing the efficacy of CT-P10 and RTX in patients with cancer are yet to be published. However, the Phase III study in advanced FL was designed to assess the noninferiority of efficacy of CT-P10 versus US-RTX. The primary efficacy end point of this ongoing study is overall response rate over the 24-week induction period, defined as the proportion of patients who achieved a complete response, an unconfirmed complete response or a partial response $[49,50]$. 
Safety

Data from the RA population show that CT-P10 and RTX share similar safety profiles. Up to week 24 of the Phase I study, adverse events occurred in 51.0 and $74.5 \%$ of patients in the CT-P10 and EU-RTX groups, respectively; serious adverse events were reported in 4.9 and $5.9 \%$ of patients [44]. Two treatmentrelated adverse events led to patient withdrawal from the study (one case of hepatic steatosis in the CT-P10 group and one case of neutropenia in the EU-RTX group). The proportion of patients experiencing infections (23.5 vs $25.5 \%$ ) and infusion reactions (16.7 vs $19.6 \%$ ) were also similar between the CT-P10 and EU-RTX groups. No grade 4 adverse events or deaths occurred in either group [44]. In the Phase III study, the proportion of patients affected by study drug-related adverse events up to week 24 were similar in the CT-P10 and RTX groups (30.4 and $28.0 \%$, respectively), as was the proportion experiencing study drug-related infection (8.1 and $10.4 \%$ ) [51]. Up to week 24 , there were no serious infusion-related reactions and no cases of malignancy or progressive multifocal leukoencephalopathy.

Data up to treatment cycle 4 of the Phase III study in advanced FL has also revealed comparable safety profiles for CT-P10 and RTX [48]. In the CT-P10 and US-RTX groups, respectively, 72.9 and $66.1 \%$ of patients experienced one or more adverse events; 3.4 and $3.2 \%$ had at least one study drug-related serious adverse event. Adverse events of special interest either occurred at similar frequencies in the CT-P10 and US-RTX groups (infusion-related reaction, 25.4 vs $21.0 \%$; infection, 20.3 vs $21.0 \%$ ) or were not observed (progressive multifocal leukoencephalopathy, serious viral infection, hepatitis B virus reactivation).

\section{Immunogenicity}

The proportion of patients in whom ADAs are produced reflects the degree of immunogenicity of a biologic drug. In the Phase I RA study, ADAs were detected in $17.6 \%$ of patients with RA in both the CT-P10 and EU-RTX groups at week 24 [44]. Stratification of patients in this study according to the presence or absence of ADAs did not reveal statistically significant effects of ADAs on the incidence of adverse events, serious adverse events, infections or infusion reactions in either treatment group. Similarly, ADAs did not interfere with efficacy within either group, as measured by ACR20 response rates and other end points [44]. In the Phase III FL study, the proportion of patients with ADAs at any post-treatment visit up to induction cycle 4 was similar in the CT-P10 and US-RTX groups (5.1 and 3.2\%, respectively) [48].

\section{Conclusion}

The escalating global economic burden of cancer requires alternative approaches to widen access to effective but high-cost biologic drugs. Less expensive biosimilar drugs, which due to the very nature of biological products cannot be identical to their reference drugs, are carefully developed and assessed to ensure they possess no clinically meaningful differences in efficacy or safety versus their reference products. Indeed, regulatory guidelines established by the EMA and FDA require robust evidence of comparability that is collected using a cumulative, stepwise approach. This approach involves collection of data in analytical, in vitro, nonclinical in vivo and clinical studies. CT-P10 is the first $\mathrm{mAb}$ biosimilar cancer therapeutic to gain approval in Europe according to these criteria, based on its biosimilarity to RTX. Consequently, important differences between these two biologic drugs are not expected to arise, although as for all new medicines, continued monitoring and pharmacovigilance will be necessary. CT-P10 is now licensed for all the same indications as RTX including, in the oncology field, chronic lymphocytic leukemia, FL and diffuse large B cell lymphoma. CT-P10 therefore sits at the vanguard of this novel approach to anticancer drug development.

\section{Future perspective}

Based on positive experiences with first-generation biosimilars in oncology supportive care, the emergence of second-generation biosimilar cancer therapeutics, typified by CT-P10, is expected to permit lower pricing and stimulate competition within the pharmaceutical marketplace, with the potential for cost savings and mitigation of geographical inequalities in access. Ultimately, however, the impact of these biosimilars will depend on their adoption by clinicians and other stakeholders. Despite some initial resistance in certain countries [52], the rapid rise in uptake of infliximab biosimilars in Europe illustrates the potential receptiveness of the clinical and economic environment to 
second-generation biosimilars [39]. Uptake of biosimilar cancer therapeutics is expected to be strengthened by clinician education on the rigorous regulatory framework in which they are developed and the accumulating body of evidence relating to the safety and efficacy of currently licensed biosimilars.
Acknowledgements

Medical writing support during the preparation of this article was provided by Hannah Mace MPharmacol and Rick Flemming, PhD, CMPP (Aspire Scientific, Bollington, UK) and was funded by CELLTRION Healthcare Co., Ltd (Incheon, Republic of Korea).

\section{EXECUTIVE SUMMARY}

\section{Background}

- Targeted biologics have helped revolutionize the treatment of cancer but are complex and expensive to produce.

- The increasing incidence of cancer, partly attributable to an aging population, is further contributing to escalating expenditure and rationing of treatment.

- Biosimilars represent a less costly alternative to originator biologics.

\section{Biosimilars: definitions \& differences versus generic drugs}

- Biologic drugs are heterogeneous in their nature, particularly with respect to post-translational modifications; therefore, biosimilars lack the chemical identity with their reference product that is associated with small-molecule generic drugs.

- Nonetheless, biosimilar drugs are developed to be highly comparable to their reference product with respect to quality, biological activity, safety and efficacy, notwithstanding minor differences in clinically inactive components. This contrasts with noncomparable biologics for which rigorous comparative data are not available.

\section{Rationale for development \& introduction of biosimilar cancer therapeutics}

- Although cost savings are not as marked as with generics, biosimilars are typically associated with discounts of 20-35\% versus their reference products.

- First-generation biosimilars used in oncology supportive care have already increased affordability and improved access, and similar benefits are expected with second-generation biosimilar cancer therapeutics.

- Large cumulative budgetary savings are predicted in Europe and the USA between 2016 and 2020 following patent expiry of key originator biologics.

\section{Scientific rationale \& regulatory requirements for biosimilar cancer therapeutics}

- Both FDA and EMA regulatory guidelines employ a stringent stepwise approach to demonstrate biosimilarity, encompassing analytical, in vitro, nonclinical in vivo and clinical comparisons with the reference product.

- Such regulatory agencies consider the 'totality of evidence' when making decisions on biosimilar approval. Comprehensive evaluation at early stages reduces the extent of subsequent data required to resolve outstanding uncertainty.

\section{Case study: development \& approval of the rituximab biosimilar CT-P10}

- CT-P10 is the first biosimilar cancer therapeutic drug to gain marketing approval and, like reference rituximab, acts by binding to the CD20 antigen and triggering B cell death.

- Licensed indications of CT-P10 are chronic lymphocytic leukemia, follicular lymphoma, diffuse large B cell lymphoma, rheumatoid arthritis, granulomatosis with polyangiitis (GPA) and microscopic polyangiitis (MPA).

- CT-P10 is highly similar to reference rituximab with respect to structure, purity, in vitro activity, in vivo toxicokinetics and clinical pharmacokinetics, pharmacodynamics, efficacy, safety and immunogenicity.

\section{Conclusion}

- Regulatory guidelines require robust evidence of comparability that is collected using a cumulative, stepwise approach.

- CT-P10 is the first mAb biosimilar cancer therapeutic to gain approval in Europe according to these criteria, based on its biosimilarity to rituximab. 


\section{Financial \& competing interests disclosure}

$C$ Buske has received research funding from Roche, CELLTRION and Janssen, and personal fees from Roche, CELLTRION, Janssen, Pfizer and Bayer outside the submitted work. M Ogura has received research funding from Symbio, and personal fees from CELLTRION, Celgene, AstraZeneca, Takeda, Mundipharma, Janssen and Meiji Seika Pharma outside the submitted work. H-C Kwon and SW Yoon are employees of CELLTRION Healthcare Co., $L t d$. The authors have no other relevant affiliations or financial involvement with any organization or entity with a financial interest in or financial confict with the subject matter or materials discussed in the manuscript apart from those disclosed.

Writing assistance was utilized in the production of this manuscript.

\section{References}

Papers of special note have been highlighted as:

- of interest; $\bullet$ of considerable interest

1 Feldmann M, Maini RN. Anti-TNF therapy, from rationale to standard of care: what lessons has it taught us? J. Immunol. 185(2), 791-794 (2010)

2 Keating GM. Rituximab: a review of its use in chronic lymphocytic leukaemia, low-grade or follicular lymphoma and diffuse large B-cell lymphoma. Drugs 70(11), 1445-1476 (2010).

3 Scott AM, Wolchok JD, Old LJ. Antibody therapy of cancer. Nat. Rev. Cancer 12(4), 278-287 (2012).

4 Strand V, Kimberly R, Isaacs JD. Biologic therapies in rheumatology: lessons learned, future directions. Nat. Rev. Drug Discov. 6(1), 75-92 (2007).

5 Kelly C, Mir F. Biological therapies: how can we afford them? BMJ 339, b3276 (2009).

6 Schellens J. Controversy of the day. Biosimilars and bioequivalents: a wise choice in a demanding treatment landscape? ESMO 2016 Daily Reporter. www.esmo.org/ Conferences/ESMO-2016-Congress/ News-Articles/Controversy-of-the-DayBiosimilars-and-bioequivalents-A-wisechoice-in-a-demanding-treatment-landscape/

7 Fromer M. Understanding biosimilars and their impending role in oncology care. ASCO Post. www.ascopost.com/issues/ november-25-2016/understandingbiosimilars-and-their-impending-role-inoncology-care/

8 European Medicines Agency. European public assessment report for Truxima. www.
ema.europa.eu/docs/en_GB/document_ library/EPAR_-_Summary_for_the_public/ human/004112/WC500222696.pdf

9 European Medicines Agency. Guideline on similar biological medicinal products containing biotechnology-derived proteins as active substance: non-clinical and clinical issues. www.ema.europa.eu/docs/en_GB/ document_library/Scientific_ guideline/2015/01/WC500180219.pdf

10 U.S. Food \& Drug Administration. Scientific considerations in demonstrating biosimilarity to a reference product. Guidance for industry. www.fda.gov/downloads/drugs/guidances/ ucm291128.pdf

-• US FDA guideline detailing current requirements for demonstrating biosimilarity to a reference product.

11 Tabernero J, Vyas M, Giuliani R et al. Biosimilars: a position paper of the European Society for Medical Oncology, with particular reference to oncology prescribers. ESMO Open 1(6), e000142 (2017).

-• European Society for Medical Oncology position statement on biosimilars and issues in the field of oncology.

12 Camacho LH, Frost CP, Abella E, Morrow PK, Whittaker S. Biosimilars 101: considerations for U.S. oncologists in clinical practice. Cancer Med. 3(4), 889-899 (2014).

13 Schellekens H, Smolen JS, Dicato M, Rifkin RM. Safety and efficacy of biosimilars in oncology. Lancet Oncol. 17(11), e502-e509 (2016).

14 Rugo HS, Linton KM, Cervi P, Rosenberg JA, Jacobs I. A clinician's guide to biosimilars in oncology. Cancer Treat. Rev. 46, 73-79 (2016).

15 European Medicines Agency. Procedural advice for users of the centralised procedure for generic/hybrid applications. www.ema. europa.eu/docs/en_GB/document_library/ Regulatory_and_procedural_ guideline/2009/10/WC500004018.pdf

16 European Medicines Agency. Guideline on the investigation of bioequivalence. www. ema.europa.eu/docs/en_GB/document_ library/Scientific_guideline/2010/01/ WC500070039.pdf

17 Francescon S, Fornasier G, Baldo P. Biosimilar oncology drugs in Europe: regulatory and pharmacovigilance considerations. Oncol. Ther. 4, 173-182 (2016).

18 Macdonald JC, Hartman H, Jacobs IA. Regulatory considerations in oncologic biosimilar drug development. MAbs 7(4), 653-661 (2015).
19 Mysler E, Pineda C, Horiuchi T et al. Clinical and regulatory perspectives on biosimilar therapies and intended copies of biologics in rheumatology. Rheumatol. Int. 36(5), 613-625 (2016).

20 Generics and Biosimilars Initiative. Biosimilars approved in Europe. www. gabionline.net/Biosimilars/General/ Biosimilars-approved-in-Europe

21 Amgen Ltd. Neupogen Summary of Product Characteristics. Neupogen $30 \mathrm{MU}(0.3 \mathrm{mg} /$ $\mathrm{ml})$ solution for injection. www.medicines. org.uk/emc/medicine/27485

22 Janssen-Cilag Ltd. Eprex Summary of Product Characteristics. Eprex 10,000 IU/ml solution for injection in pre-filled syringe. www.medicines.org.uk/emc/medicine/29446

23 Sandoz Inc. Zarxio Prescribing Information. www.accessdata.fda.gov/drugsatfda_docs/ label/2015/125553lbl.pdf

24 CELLTRION Inc. Inflectra Prescribing Information. www.accessdata.fda.gov/ drugsatfda_docs/label/2016/125544s000lbl. pdf

25 CELLTRION Inc. Remsima Summary of Product Characteristics. www.ema.europa.eu/ docs/en_GB/document_library/EPAR_-_ Product_Information/human/002576/ WC500150871.pdf

26 U.S. Food \& Drug Administration. List of licensed biological products with (1) reference product exclusivity and (2) biosimilarity or interchangeability evaluations to date. www. fda.gov/downloads/Drugs/ DevelopmentApprovalProcess/ HowDrugsareDevelopedandApproved/ ApprovalApplications/

TherapeuticBiologicApplications/Biosimilars/ UCM439049.pdf

27 American Cancer Society. LIVESTRONG. The global economic cost of cancer. https:// old.cancer.org/acs/groups/content/@ internationalaffairs/documents/document/ acspc-026203.pdf

28 World Health Organization. Early cancer diagnosis saves lives, cuts treatment costs. www.who.int/mediacentre/news/ releases/2017/early-cancer-costs/en/

29 Cornes P. The economic pressures for biosimilar drug use in cancer medicine. Target Oncol. 7(Suppl. 1), S57-S67 (2012).

30 Mariotto AB, Yabroff KR, Shao Y, Feuer EJ, Brown ML. Projections of the cost of cancer care in the United States: 2010-2020. J. Natl Cancer Inst. 103(2), 117-128 (2011).

31 I.M.S. Health. Global market for cancer treatments grows to $\$ 107$ billion in 2015 , fuelled by record level of innovation. www. 
imshealth.com/en/about-us/news/ims-healthstudy-global-market-for-cancer-treatmentsgrows-to-107-billion-in-2015-fueled-byrecord-level-of-innovation

32 I.M.S. Institute for Healthcare Informatics. Medicines use and spending in the U.S. A review of 2015 and outlook to 2020. https:// morningconsult.com/wp-content/ uploads/2016/04/IMS-Institute-US-DrugSpending-2015.pdf

33 Jacobs I, Ewesuedo R, Lula S, Zacharchuk C. Biosimilars for the treatment of cancer: a systematic review of published evidence. BioDrugs 31(1), 1-36 (2017).

- Systematic review of all published clinical and nonclinical studies of proposed biosimilars and intended copies ('biomimics') for the treatment of cancer.

34 Socinski MA, Curigliano G, Jacobs I, Gumbiner B, MacDonald J, Thomas D. Clinical considerations for the development of biosimilars in oncology. MAbs 7(2), 286-293 (2015).

35 Gori S, Di Maio M, Pinto C et al. Differences in the availability of new anti-cancer drugs for Italian patients treated in different regions. Results of analysis conducted by the Italian Society of Medical Oncology (AIOM). Tumori 96(6), 1010-1015 (2010).

36 Bennett CL, Chen B, Hermanson T et al. Regulatory and clinical considerations for biosimilar oncology drugs. Lancet Oncol. 15(13), e594-e605 (2014).

37 I.M.S. Institute for Healthcare Informatics. Delivering on the potential of biosimilar medicines. The role of functioning competitive markets. www.imshealth.com/ files/web/IMSH\%20Institute/ Healthcare\%20Briefs/Documents/IMS Institute_Biosimilar_Brief_March_2016.pdf

38 Brodszky V, Baji P, Balogh O, Pentek M. Budget impact analysis of biosimilar infliximab (CT-P13) for the treatment of rheumatoid arthritis in six Central and Eastern European countries. Eur. J. Health Econ. 15(Suppl. 1), S65-S71 (2014).

39 Han S, Kim S, Kim J et al. The pharmacoeconomic impact of biosimilar infliximab (CT-P13) in Europe from January 2015 to June 2016. Presented at: 12th Congress of the European Crohn's and Colitis
Organisation. Barcelona, Spain, 15-18 February 2017 (Abstract P582).

40 European Medicines Agency. Guideline on similar biological medicinal products containing biotechnology-derived proteins as active substance: quality issues (revision 1). www.ema.europa.eu/docs/en_GB/ document_library/Scientific_ guideline/2014/06/WC500167838.pdf

41 European Medicines Agency. Guideline on similar biological medicinal products containing monoclonal antibodies - nonclinical and clinical issues. www.ema.europa. eu/docs/en_GB/document_library/ Scientific_guideline/2012/06/ WC500128686.pdf

- EMA guideline detailing the well-defined regulatory requirements for approval of a biosimilar, involving rigorous evaluations and comparisons with its reference product.

42 U.S. Food \& Drug Administration. Quality considerations in demonstrating biosimilarity of a therapeutic protein product to a reference product. Guidance for industry. www.fda. gov/downloads/drugs/guidances/ucm291134. pdf

43 Cartron G, Watier H, Golay J, Solal-Celigny P. From the bench to the bedside: ways to improve rituximab efficacy. Blood $104(9)$, 2635-2642 (2004).

44 Yoo D-H, Suh C-H, Shim SC et al. A multicentre randomised controlled trial to compare the pharmacokinetics, efficacy and safety of CT-P10 and innovator rituximab in patients with rheumatoid arthritis. Ann. Rheum. Dis. 76(3), 566-570 (2017).

- Phase I study of CT-P10 compared with EU-sourced reference rituximab in rheumatoid arthritis demonstrating pharmacokinetic equivalence and comparable efficacy, pharmacodynamics, safety and immunogenicity.

45 Clinical Trials Database: NCT01534884. https://clinicaltrials.gov/ct2/show/ NCT01534884

46 Clinical Trials Database: NCT02149121. https://clinicaltrials.gov/ct2/show/ NCT02149121

47 Suh C-H, Kasay A, El-Khouri E et al. Pharmacokinetics and safety of three formulations of rituximab (CT-P10, US-sourced innovator rituximab and EU-sourced innovator rituximab) in patients with rheumatoid arthritis: results from Phase 3 randomized controlled trial over 24 weeks. Arthritis Rheumatol. 68(Suppl. 10), Abstract 1634 (2016).

- Phase III study of CT-P10 compared with US- and EU-sourced reference rituximab in rheumatoid arthritis demonstrating pharmacokinetic equivalence and comparable safety.

48 Coiffier B, Sancho J-M, Jurczak W et al. Pharmacokinetic and safety of CT-P10, a biosimilar candidate to the rituximab reference product, in patients with newly diagnosed advanced stage follicular lymphoma. Blood 128(22), Abstract 1807 (2016).

- Phase III study of CT-P10 compared with US-sourced reference rituximab in patients with advanced follicular lymphoma plus chemotherapy, demonstrating pharmacokinetic equivalence and comparable safety and immunogenicity.

49 Clinical Trials Database: NCT02162771. https://clinicaltrials.gov/ct2/show/ NCT02162771

50 Kim W, Ogura M, Buske C et al. Global phase I/III pharmacokinetic and efficacy study comparing CT-P10, a biosimilar candidate to the rituximab reference product in patients with advanced stage follicular lymphoma (AFL). Blood 126(23), 5111 (2015).

51 Yoo D-H, Majstorovic L, Kasay A et al. Efficacy and safety of CT-P10, rituximab biosimilar candidate, and innovator rituximab in patients with rheumatoid arthritis: results from Phase 3 randomized controlled trial over 24 weeks. Arthritis Rheumatol. 68(Suppl. 10), Abstract 1635 (2016).

- Phase III study of CT-P10 compared with US- and EU-sourced reference rituximab in rheumatoid arthritis demonstrating therapeutic equivalence and comparable pharmacodynamics and safety.

52 Braun J, Kudrin A. Switching to biosimilar infliximab (CT-P13): evidence of clinical safety, effectiveness and impact on public health. Biologicals 44(4), 257-266 (2016). 